\title{
A INFLUÊNCIA DOS VALORES ORGANIZACIONAIS NA PERCEPÇÃO DE POLÍTICAS E PRÁTICAS DE GESTÃo DE PESSOAS
}

\author{
Gisela Demo* \\ giselademo@unb.br \\ Thais Fernandes* \\ Thais.frnnds@hotmail.com \\ Natasha Fogaça* \\ Natasha.adm@gmail.com \\ *Universidade de Brasília
}

http://dx.doi.org/10.1590/1413-2311.093.57040

Recebido em 11/07/2015

Aprovado em 16/02/2017

Disponibilizado em 31/05/2017

Avaliado pelo sistema "doubleblindreview"

Revista Eletrônica de Administração

Editora-chefe: Andrea Oltramari

ISSN 1413-2311 (versão “online”)

Editada Pela Escola de Administração da Universidade Federal do Rio Grande do Sul

Periodicidade: Quadrimestral

Sistema requerido: Adobe Acrobat Reader

\section{RESUMO}

Nota-se que os valores organizacionais possuem uma relação direta com as práticas das organizações, dentre elas, as de gestão de pessoas, pois ambos formam o núcleo da cultura decada organização. Por conseguinte, o objetivo geral deste estudo foi analisar a relação existente entre valores organizacionais, como variável independente, e políticas e práticas de gestão de pessoas, como variável dependente. A pesquisa pode ser classifica como teóricoempírica quanto ao seu enquadramento, e quantitativa quanto à sua natureza. A pesquisa foi aplicada em uma empresa pública do ramo de agricultura do Distrito Federal, utilizando questionários aplicados online e presencialmente. Quanto às análises utilizadas, foi realizado Escalonamento Multidimensional (MDS) para os dados de valores organizacionais, e regressões múltiplas lineares para analisar a relação existente entre as duas variáveis. Além disso, foi realizada uma revisão bibliométrica para as duas variáveis no período de 2009 a 
2014, analisando os periódicos científicos de primeira linha e a institucionalização das pesquisas no Brasil para cada uma das variáveis foi traçada.

Palavras-Chave:Gestão de Pessoas;Políticas e Práticas de Gestão de Pessoas; Valores Organizacionais; Estudo Relacional; Empresa Pública.

\title{
THE INFLUENCEOF ORGANIZATIONAL VALUES IN THE PERCEPTION OF HUMAN RESOURCE MANAGEMENT POLICIES E PRACTICES
}

\begin{abstract}
The organizational values have a direct relationship with the practices of organizations, among them, Human Resource Management (HRM), as they both form the core of the culture of each organization. Therefore, the aim of this study was to analyze the relationship between organizational values, as an independent variable, and HRM policies, as the dependent variable. The research can be classified as theoretical and empirical as its framework, and quantitative as to its nature. The survey was conducted in anagricultural public organizationlocated at Distrito Federal, using online questionnaires and in person. For analyzes, it was conducted Multidimensional Scaling (MDS) for organizational values data, and multiple linear regressions to analyze the relationship between the two variables. In addition, a bibliometric review for the two variables in the period 2009 to 2014 was conducted to analyze the scientific journals of the first line and the institutionalization of research in Brazil for each of the variables was drawn.
\end{abstract}

Keywords: Human Resource Management;HRM Policies and Practices;Organizational Values;Relational Study;Public Organization

\section{LA INFLUENCIA DE LOS VALORES ORGANIZACIONALES EN LA PERCEPCION DE LAS POLÍTICAS Y PRÁCTICAS DE GESTIÓN DE PERSONAS}

\section{RESUMEN}

Tenga en cuenta que los valores organizacionales tienen una relación directa con las prácticas de las organizaciones, entre ellas, la gestión de personas, ya que ambos forman el núcleo de la 
cultura de cada organización. Por lo tanto, el objetivo de este estudio fue analizar la relación entre los valores organizacionales, como una variable independiente, y las políticas y prácticas de gestión de personas, como la variable dependiente. La investigación puede ser clasificada como teórica y empírica como marco, y cuantitativo en cuanto a su naturaleza. La encuesta se realizó en una empresa pública en la rama agrícola del Distrito Federal, a través de cuestionarios online y en persona. En cuanto a los análisis utilizados, que se llevó a cabo el Escalamiento Multidimensional (MDS) para los datos de los valores organizacionales, y regresion lineal múltiple para analizar la relación entre las dos variables. Además, una revisión bibliométrica de las dos variables en el período 2009-2014 se llevó a cabo mediante la revisión de las revistas científicas de la primera línea y la institucionalización de la investigación en Brasil para cada una de las variables fue dibujado.

Palabras Clave: Gestión de Personas; Políticas y Prácticas en la Gestión de Personas; Valores Organizacionales; Estudio Relacional; Empresa Pública.

\section{INTRODUÇÃO}

O cenário do novo quadro competitivo, constituído por novas formas de concorrência, mutabilidade, inovação e crescente valorização do conhecimento, fizeram com que as organizações e a Gestão de Pessoas (GP), ou Gestão de Recursos Humanos (GRH), se reestruturassem (SANTOS, 2004). Segundo o autor, os modelos de GP são a forma como a organização se organiza frente ao gerenciamento do comportamento humano; esses modelos sofrem modificações constantes para se adequar às novas realidades. Um dos atuais objetivos da GP é diferenciar a organização frente ao seu mercado, gerando uma contribuição positiva na imagem e na competitividade.

As políticas e práticas de GP deixaram de ser vistas sob o aspecto de mais um custo para a organização e passaram a ser vistas como uma fonte de vantagem competitiva, uma vez que membros motivados e alinhados com os objetivos organizacionais se tornam um dos ativos mais valiosos para as organizações no atual ambiente mercadológico (OLIVEIRA; OLIVEIRA, 2011).

A Gestão de Pessoas em sua versão mais atual, e consequentemente suas políticas e práticas, entende o colaborador como fator estratégico, uma vez que que ele passa a ser a peça 
chave para o alcance de efetividade e competitividade organizacional cada vez maiores (HORTA; DEMO; ROURE, 2012). As políticas de GP se operacionalizam por meio de suas práticas e se relacionam ainda com os valores, expectativas e posicionamento organizacional, além de servirem como referência para práticas organizacionais e possíveis decisões tomadas pelos membros inseridos nas mesmas (ARMSTRONG, 2012).

Dentro dos estudos da psicologia organizacional, a cultura organizacional mostra-se um tema de grande importância, uma vez que é considerada como fator determinante para o desempenho individual, a satisfação do funcionário e a produtividade da empresa (TAMAYO; MENDES; PAZ, 2000). Segundo os autores, neste contexto de cultura organizacional, surgem os valores, que norteiam o comportamento do indivíduo. Para Tamayo e Borges (2006), os valores organizacionais são definidos como princípios ou crenças, que são organizados hierarquicamente, relacionados a condutas e metas desejáveis, que acabam servindo de orientação para a vida grupal, podendo satisfazer os interesses pessoais, coletivos ou ambos.

Nota-se que os valores organizacionais possuem uma relação direta com as práticas das organizações, pois ambos formam o núcleo da cultura de cada organização (HOFSTEDE et al., 1990). As práticas organizacionais, na qual as de GP se incluem, podem ser definidas como padrões seguidos pelos funcionários para realizar suas atividades de trabalho, e também os procedimentos utilizados nas relações entre funcionários e seus clientes (GUARDANI et al., 2013). Por conseguinte, os valores organizacionais influenciam os comportamentos que por consequência influenciam as práticas existentes na organização.

Dessa forma, o presente estudo visa estudar as relações entre os valores organizacionais e as políticas e práticas de gestão de pessoas, enquadradas dentro de seis grandes políticas, conforme modelo proposto por Demo, Neiva, Nunes, Rozzett (2012): Recrutamento e Seleção; Envolvimento; Treinamento, Desenvolvimento e Educação; Condições de Trabalho; Recompensas e Remuneração e Avaliação de Desempenho baseada em Competências.

\section{VALORES ORGANIZACIONAIS}

A cultura organizacional é um tema de grande importância para os estudos da psicologia organizacional, uma vez que está relacionada com o desempenho individual, satisfação no trabalho e produtividade (TAMAYO; MENDES; PAZ, 2000). Nos últimos anos, 
o interesse por estudos de valores organizacionais cresceu, uma das explicações para este acontecimento é o seu papel fundamental para o entendimento da cultura (CARVALHO; OLIVEIRA; SILVA, 2013).

Os valores, juntamente com os papéis e as normas, representam um dos principais componentes de uma organização, uma vez que definem e orientam o comportamento da mesma (OLIVEIRA; TAMAYO, 2004). Segundo Tamayo e Borges (2006), os valores podem ser definidos como princípios ou crenças que são organizados de forma hierárquica, relativos às metas ou condutas desejáveis e que orientam a vida em grupo, podendo se relacionar aos interesses individuais, do grupo ou de ambos. Uma vez que os valores são compartilhados por todos ou pela grande maioria dos membros da organização, eles possuem ainda uma função integradora (OLIVEIRA; TAMAYO, 2004).

Nesse sentido, Veiga (2010) argumenta que os valores organizacionais não são os valores pessoais dos integrantes da organização. Os valores pessoais são aprendidos e desenvolvidos durante o convívio familiar, escolar e nas interações sociais ao longo da existência do indivíduo, enquanto os valores organizacionais são geralmente introduzidos pelo dono ou criador da empresa ou por indivíduos que exercem forte influência no ambiente organizacional.

Os valores têm origem na interação social e são construídos com base nas necessidades de trabalho e para orientar as relações existentes na organização, eles atuam como elementos que definem, especificam e tornam uma organização singular, diferente de todas as outras existentes (HASSAN, 2007).

Uma importante teoria sobre o tema, a Teoria de Valores Culturais de Schwartz (1999), defende que os valores culturais representam problemas básicos enfrentados pela sociedade, sendo eles: definir a natureza da relação entre grupo e indivíduo; estabelecer comportamento responsável que preserve o grupo social; e definir a relação das pessoas com o mundo social e natural. Através dessas necessidades, emergem três dimensões bipolares de valores: Autonomia X Conservadorismo; Hierarquia X Igualitarismo; Domínio X Harmonia. Foram realizados testes com as dimensões propostas para validar o modelo em 49 nações, o que o tornou universal.

Tamayo, Mendes e Paz (2000) adaptaram a teoria proposta por Schwartz (1999) para a realidade organizacional, no entanto, o estudo apresentou a falta de oposição entre alguns pólos das dimensões propostas. Assim, com o objetivo de realizar uma nova investigação 
sobre o assunto, Porto et al. (2013) tomaram por base o modelo proposto e testado por Schwartz (1999). Os dados obtidos se mostraram satisfatórios, mostrando que a teoria utilizada é adequada para o estudo de valores organizacionais. Um pólo foi alterado, Autonomia x Conservadorismo passa a ser Inovação x Estabilidade, uma vez que a dimensão Igualitarismo pode englobar Autonomia no quesito de buscas de suas próprias ideias, e que há uma mudança de nível de análise dos valores organizacionais e o foco passa a ser a capacidade de a organização buscar inovação.

Em suma, os valores têm como função orientar a organização e guiar comportamentos e são de extrema importância para o dia-a-dia da empresa. A diferenciação pessoal, organizacional e cultural está baseada principalmente pela hierarquia dos valores, eles podem até ser iguais, mas ao serem organizados e priorizados de formas diferentes, se tornam diferentes. Implicam necessariamente em preferência, o que virá como prioritário e o que será alocado após isso (TAMAYO; GONDIM, 1996). Os valores são tão importantes que sua percepção por partes dos membros impactam diretamente a rotina organizacional e o comprometimento existente entre empresa e funcionário (TAMAYO; MENDES; PAZ, 2000). Por isso, eles devem ser analisados e trabalhados cuidadosamente.

Com vistas a se obter um panorama do cenário de produção nacional, foi realizado um levantamento bibliográfico sobre Valores Organizacionais abrangendo um período de 6 anos, de 2009-2014. Foram pesquisados os periódicos científicos brasileiros de primeira linha, ou seja, classificados com avaliação maior ou igual a B1 pelo sistema Qualis da Coordenação de Aperfeiçoamento de Pessoal de Nível Superior - CAPES da área de Administração e Psicologia.

No total, foram encontrados 31 artigos. A Revista de Administração Mackenzie foi o periódico que mais publicou artigos sobre o tema, com uma representatividade de $16 \%$. Dos artigos analisados, $97 \%$ se classificaram como teórico-empíricos, sendo que $73 \%$ deles eram quantitativos e $40 \%$ realizaram suas pesquisas na esfera privada. As instituições de ensino que mais publicaram sobre o tema foram a Universidade Presbiteriana Mackenzie e a Universidade de Brasília, cada uma com $16 \%$ da publicação total.

No que se refere à institucionalização da pesquisa de Valores Organizacionais, um retrato atual pode ser obtido a partir dos grupos de pesquisa identificados com a denominação "valores organizacionais". 
O Diretório de Grupo de Pesquisa - Base Corrente - da Plataforma Lattes, revela a existência de 7 grupos para a busca "valores organizacionais", dos quais 1 não se enquadra nesta pesquisa por se tratar de valores pessoais. Assim, os grupos relacionados a "valores organizacionais" totalizam 6 , dos quais três (ou 50\%) pertencem à área de administração, e três (ou 50\%) pertencem à área de psicologia.

\section{POLÍTICAS E PRÁTICAS DE GESTÃo DE PESSOAS}

No que tange à evolução dos estudos relativos à GP no Brasil, o trabalho de Wood Jr., Tonelli e Cooke (2011) o qual analisou o período de 1980 a 2010, mostrou que a área estava mais focada em alinhar suas políticas com o novo cenário que emergia marcado pela abertura de mercado, o surgimento do empreendedorismo, o aumento da concorrência e a busca pela inovação, qualidade e eficiência, visando principalmente incluir o Brasil nesse ambiente. Neste contexto, a atração, capacitação, valorização e retenção de talentos assume conotação especial, constituindo o principal desafio estratégico da GP no novo milênio, delineando os pressupostos, fundamentos e conceitos da moderna GP.

Assim, considerando os conceitos, fundamentos e pressupostos da gestão de pessoas, de acordo com Mascarenhas e Kirschbaum (2008), a emergência da gestão estratégica de pessoas, associada à tese do alinhamento estratégico propiciou o surgimento de novas ferramentas de GP úteis à construção de sistemas produtivos que valorizem o envolvimento, a integração e o desenvolvimento dos indivíduos. Sob esta perspectiva, Armstrong (2009) argumenta que a GP deve ser coerente com os mais primordiais valores da organização sendo suas principais características a estratégia com ênfase na integração; o foco no empreendimento e nos valores do negócio; e a base na crença de que as pessoas devem ser tratadas como recursos valiosos para a organização (capital humano). Similarmente, na visão de Bohlander e Snell (2009), a gestão de recursos humanos é o processo de gerenciar talentos humanos para atingir os objetivos da organização.

Mathis e Jackson (2003), por seu turno, definem a GP como a habilidade de gerenciar os recursos humanos, por meio de políticas e práticas, para conquistar e manter vantagem competitiva e, assim, as pessoas constituem a principal core competence, ou competência essencial, das organizações. Conforme os autores, uma competência essencial pode ser definida como a capacidade organizacional que cria alto valor e que diferencia a organização 
de seus concorrentes. Segundo Mascarenhas (2009), uma competência essencial possui três características: é valiosa e versátil, podendo ser utilizada em uma ampla variedade de mercados; oferece reais benefícios aos consumidores; e é de difícil imitação.

Conforme Guest (1987), os fundamentos da GP envolvem o objetivo de integração, isto é, os recursos humanos integrados aos planos estratégicos da organização; o objetivo de comprometer os empregados com a organização; o objetivo de flexibilidade/adaptabilidade das estruturas e funções organizacionais; e o objetivo de qualidade, isto é, qualidade em relação às pessoas, ao desempenho, aos padrões e à imagem pública.

A partir do pressuposto de que a GP precisa ser alçada ao nível estratégico das organizações, Legge $(1995,2006)$ conceitualiza a GP estratégica (StrategicHumanResource Management, no inglês) como a gestão dos relacionamentos com os colaboradores para contribuir o máximo possível com a consecução das metas organizacionais e ressalta dois pontos importantes para a GP: as políticas de GP devem ser integradas ao planejamento estratégico da organização e usadas para reforçar uma cultura organizacional apropriada; e os recursos humanos constituem uma valiosa fonte de vantagem competitiva.

Neste contexto, a moderna GP distingue-se da clássica Administração de Pessoal. Legge (1995) propõe que, em se tratando de gestão de recursos humanos, as teorias podem ser agrupadas em duas versões: hard (dura ou conservadora) e soft (macia ou moderna). A versão hard foca principalmente a questão da integração das políticas de RH com a estratégia empresarial, com ênfase nos aspectos quantitativos e calculativos, entendendo as pessoas como qualquer outro recurso econômico. Seria propriamente a gestão de recursos humanos (grifo da autora). E a versão soft, de acordo com Legge (1995), além de primar pela integração de atividades de RH e estratégias empresariais, entende as pessoas como patrimônio organizacional valioso e fonte de vantagem competitiva. Os empregados passam a ser insumos proativos do processo produtivo e não meros recursos passivos. O que se pretende, nesta versão, é gerar comprometimento via comunicação, liderança e motivação. Nesse caso, fala-se de gestão de recursos humanos (grifo da autora).

Legge (1995) afirma que, mais que uma perspectiva diferenciada (de melhor tratamento das pessoas) da clássica administração de pessoal, a GP é realmente distinta por ser uma atividade mais central de gestão estratégica que a administração de pessoal, já que é vivenciada pelos gerentes e pessoas como o mais importante recurso organizacional a ser gerenciado, envolvendo-os no alcance das metas da organização. Em suma, a GP representa a 
descoberta da administração de pessoal pelos principais executivos da organização (LEGGE, 1995). Assim, nascem novos papéis, desafios e perspectivas para a GP.

No que tange ao papel da GP nas organizações atualmente, vários autores o entendem como estratégico (GUEST, 1987; STOREY, 1995; LEGGE, 2006; BOHLANDER; SNELL, 2009) defendendo que sua gestão deve ser devidamente suportada por teorias coesas e consistentes, além da imprescindibilidade de estar alinhada ao planejamento e à estratégia organizacional. Ulrichet al. (1991) afirmam que o panorama competitivo está constantemente se alterando, demandando novos modelos de competitividade que, por sua vez, exigem capacidades organizacionais que possibilitarão às empresas atenderem melhor a seus clientes e se diferenciarem de seus concorrentes. Estas capacidades organizacionais derivam da redefinição e redistribuição das práticas, funções e profissionais de GP. Por conseguinte, a GP não deve mais ter papel tradicional de suporte, mas, sim, constituir competência essencial no alcance dos objetivos e resultados organizacionais e individuais.

Do ponto de vista da GP estratégica, políticas e práticas podem ser mutuamente reforçadas para ajudar no alcance dos objetivos organizacionais (MORRIS; SNELL, 2010). Além disso, as políticas de GP precisam ser guiadas pela lógica de competências desenvolvidas em conformidade com os requisitos dos processos de negócios (SERPELL; FERRADA, 2007). Assim, elas fornecem ferramentas para capturar e comunicar a visão estratégica e os objetivos da organização em termos claros que podem ser mais facilmente entendidos e executados pelos colaboradores (VAKOLA; SODERQUIST; PRATASCOS, 2007).

Nesse sentido, Legge (2006) percebe a GP como uma nova perspectiva em gestão de pessoas, mais estratégica, e que impacta a performanceorganizacional e a experiência de trabalho dos colaboradores, assumindo patente relevância para as empresas. Para a autora, a GP estratégica estuda como as relações de trabalho são gerenciadas pela organização de forma a alcançar suas metas. A proposta de Legge (2006) está de acordo com a visão baseada em recursos da firma apresentada por Barney (1991), a qual defende que os talentos e suas interações são recursos valiosos na produção de conhecimento e consecução dos objetivos organizacionais, premissas estas que definem os pressupostos e fundamentos das políticas e práticas de GP.

Em um esforço para diferenciar esses conceitos, Martín-Alcázar, Romero-Fernández e Sánchez-Gardey (2005) argumentam que as estratégias de GP definem as diretrizes para a 
gestão da força de trabalho, já as políticas, por sua vez, buscam coordenar as práticas para que tenham coerência e sigam na mesma direção. Por fim, as práticas estão no menor nível entre os três e representam as ações de fato.

Quanto às políticas de GP, na visão de Armstrong (2009), elas definem o posicionamento, as expectativas e os valores da organização quando se trata da forma de tratamento dos indivíduos e delas derivam as principais ações dos gestores ao se depararem com problemas na área de GP. Servem, ainda, como ponto de referência para o desenvolvimento de práticas organizacionais e para decisões tomadas pelas pessoas, além de promover um tratamento equitativo entre os indivíduos. Tendo como base os fundamentos e pressupostos por ora traçados, é possível definir políticas e práticas de GP.

Em relação ao termo política organizacional, esta pode ser definida como o estabelecimento de princípios para conduta de uma empresa, um curso geral de ação no qual certas práticas são trabalhadas em conjunto, de maneira construtiva, para atingir determinados objetivos (SINGAR; RAMSDEN, 1972). Ou, ainda, a arte do relacionamento criativo de interesses conflitantes (OSBORN; HUNT; SCHERMERHORN, 1998). Não obstante, não há consenso quanto aos termos utilizados, uma vez que foram observadas nomenclaturas diversas na literatura que se referem, direta ou indiretamente, a políticas de GP, tais quais, processos, atividades, sistemas, funções e técnicas de GP.

Neste trabalho, optou-se por utilizar o termo "política" de GP, em consonância com autores como Singar e Ramsden (1972), Guest (1987), Legge (1995, 2006), Dessler (2002) e Mathis e Jackson (2003), na acepção de proposta articulada da organização, com construções teóricas e práticas, no trato das relações humanas, com vista à obtenção de resultados desejados. Dessa forma, as políticas de GP definem o referencial teórico e prático construído para possibilitar a consecução dos objetivos e finalidades da organização, funcionando como guias de pensamento e ação para a área de GP. E utilizar-se-á o termo práticas na acepção de hábito, rotina, ação, ou, ainda, atividades componentes das políticas.

As políticas e práticas de GP aqui consideradas tiveram como base a síntese do estado da arte construído por Demo (2012) e os trabalhos de Demo e Rozzett (2012) e Demo et al. (2014) para desenvolver e validar a Escala de Políticas e Práticas de Recursos Humanos. No processo de desenvolvimento e validação da escala foram realizadas entrevistas, e posterior análise de conteúdo categórica temática baseada em Bardin (2011), com cerca de 60 colaboradores de 17 organizações distintas para melhor compreender a visão deles em 
contextos organizacionais diversos quanto às políticas de GP consideradas mais importantes, adequadas e eficazes para a promoção do seu bem-estar, valorização e realização profissional e pessoal. As categorias que emergiram da análise de conteúdo foram assim denominadas, num total de 6: 1) recrutamento e seleção; 2) envolvimento; 3) treinamento, desenvolvimento e educação; 4) condições de trabalho; 5) avaliação de desempenho e competências; e 6) recompensas.

Conforme Legge (2006), a GP estratégica estuda como as relações de trabalho são gerenciadas pela organização de forma a alcançar suas metas. Tais relações de trabalho formalizam-se por meio de políticas e práticas. Nessa linha, Inyang e Akaegbu (2014) argumentam que os profissionais de RH facilitam o desenvolvimento de alta performance e de uma força de trabalho competente ao serem os responsáveis por desenhar e implementar estratégias de GP para nortear a prestação de serviços e aumentar a efetividade organizacional.

Nesse âmbito, é importante conhecer os resultados de políticas e práticas de GP constatados empiricamente por estudos científicos relacionais. Neste sentido, Uysal (2012) encontrou fortes correlações positivas e significativas entre as principais políticas de GP citadas na literatura como recrutamento e seleção, treinamento, avaliação de desempenho e remuneração. Esse resultado é importante para a compreensão das inter-relações entre políticas de GP a fim de reforçar seu efeito nos resultados organizacionais apresentados pelos empregados. De fato, algumas pesquisas evidenciaram relações positivas entre políticas de GP e variáveis como comprometimento, produtividade, lucratividade, qualidade, dentre outras (SCHNEIDER; BOWEN, 1985; GUEST, 1987; ULRICHet al., 1991) e a produção internacional recente ratifica estes resultados.

Combs et al. (2006) realizaram uma meta-análise, constatando que as relações entre práticas de GP e resultados organizacionais são mais fortes em indústrias comparativamente a empresas de serviços. Estudos também têm sido realizados em culturas diferentes da americana e da europeia. O estudo de Demo (2010) mostrou relação positiva e forte entre políticas de GP e justiça organizacional em organizações brasileiras públicas e privadas. Majumder (2012) verificou forte relação entre práticas de GP e satisfação dos empregados em bancos privados de Bangladesh. Kim e Lee (2012), ao estudarem empresas de consultoria na Coreia do Sul, encontraram evidências de que as políticas e práticas de GP melhoram tanto as capacidades estratégicas quanto o desempenho organizacional. Jia, Shaw, Tsui e Park (2014) 
desenvolveram uma perspectiva sócio-estrutural quanto às relações de trabalho, as quais são efetivadas por políticas e práticas de GP, ao mostrarem por meio de estudos empíricos em organizações de tecnologia da China, que tais relações estão associadas à criatividade das equipes de trabalho, e ainda que esta associação é mediada pela densidade de comunicação relatada no ambiente de trabalho. Ainda, Aguzolli e Geary (2014) estudaram a transferência de práticas de GP de uma multinacional brasileira para atuar no mercado canadense, revelando a habilidade em capturar componentes significativos do ambiente institucional do país anfitrião de uma forma que lhe deu o espaço necessário para propor se adaptar de maneira efetiva.

Outras pesquisas atestam que políticas e práticas de GP afetam favoravelmente a performance das organizações (HUSELID; JACKSON; SCHULER, 1997; BOSELIE; DIETZ; BOONE, 2005; SUBRAMONY, 2009; MENEZES; WOOD; GELADE, 2010). Guest e Conway (2011) confirmaram a associação entre a adoção de mais práticas de GP, maior efetividade organizacional e melhorias em uma série de indicadores de desempenho. Gomide Jr. e Tanabe (2012) também encontraram correlação entre práticas de GP e efetividade organizacional. Além disso, ALDamoe, Yazam e Ahmid (2011) concluíram que a retenção de funcionários media a relação entre práticas de GP e desempenho organizacional. As percepções dos funcionários quanto às políticas e práticas de GP também influenciam tanto o esforço de trabalho discricionário quanto a assistência ao colega de trabalho (FRENKEL, RESTUBOG; BEDNALL, 2012). Por outro lado, a efetividade e a aceitação das políticas de GP estão relacionadas aos valores e à cultura organizacional (STONE; STONE-ROMERO; LUKASZEWSKI, 2007).

Destarte, há um consenso de que as práticas de GP produzem desempenho organizacional superior quando são utilizadas em conjunto e de maneira integrada à estratégia de negócios (GUEST; HOQUE, 1994; EZZAMEL; LILLEY; WILLMOTT, 1996). E isso também é válido para pequenas empresas. O estudo conduzido por Katou (2012) mostrou que políticas e práticas de GP têm um efeito positivo sobre o desempenho organizacional avaliado por meio de atitudes (satisfação, comprometimento, motivação) e comportamentos (faltas, volume de negócios, disputas) dos empregados. Similarmente, Cantarello, Filippini e Nosella (2012) encontraram relação significativa entre as práticas de GP e a satisfação dos clientes por meio da oferta de produtos de alta qualidade. 
Ademais, estudos mostraram que as políticas de GP influenciam tanto as relações de confiança nas organizações (GOULD-WILLIAMS, 2003; TZAFRIR, 2005; MARIOTTI \& SOUZA, 2009) quanto o bem-estar no trabalho (GELADE \& IVIRY, 2003; NISHII, LEPAK \& SCHNEIDER, 2008; TURNER, HUEMANN \& KEEGAN, 2008; RUBINO, DEMO \& TRALDI, 2011).

A partir dos resultados destes estudos empíricos, parece possível afirmar que um conjunto holístico de políticas e práticas profícuas de GP devidamente sincronizado com a estratégia empresarial realmente impacta o desempenho da organização, quer seja pública ou privada, considerando resultados importantes, independentemente de como sejam medidos.

\section{MÉTODO}

A pesquisa proposta em relação aos seus objetivos se classifica como descritiva, quantitativa quanto à sua natureza, survey quanto aos meios e em relação ao corte temporal, se classifica como transversal.

A aplicação da pesquisa foi realizada em uma empresa pública do Distrito Federal criada em 1978, cuja divulgação do nome não foi autorizada. Seu ramo de atuação da é o da agricultura, e sua missão é apoiar os produtores e trabalhadores rurais do Distrito Federal. O total de funcionários que trabalham na empresa é de 298.

Em relação à amostra, esta pode ser caracterizada como não probabilística por conveniência. Uma vez que o método de análise de dados utilizado na pesquisa foi a regressão linear, é necessário obter uma amostra com um poder estatístico superior a 0,80 para as ciências comportamentais (COHEN, 1992). Foi delimitada uma amostra mínima de 97 questionários a serem preenchidos por meio dos instrumentos de pesquisa, esse número foi definido através do software GPower 3.1 que calcula o número adequado, seguindo o número de fatores abordados na variável independente dos modelos - no caso desta pesquisa, o número de fatores é 6 , considerando o critério de significância $(\alpha)$ igual a 0,05 , efeito médio do tamanho da população (ES) igual a 0,15 e $80 \%$ de poder estatístico.

Inicialmente, foram obtidos 100 questionários respondidos voluntariamente de forma presencial. Primeiramente, foi realizada a análise dos dados através distribuição de frequências (média, variância, máximo, mínimo e desvio padrão), demonstrando que os dados 
estavam coerentes.Após isso, foi realizado o tratamento de missingvalues (dados faltantes); como representavam menos do que 5\% do total, foram substituídos pela média e utilizados nas demais análises (TABACHNICK, FIDELL; 2013).

Na terceira análise, realizou-se a verificação de outliers (valores discrepantes), que correspondem a valores atípicos por se distanciarem significantemente dos dados coletados, o que pode enviesar os resultados do estudo uma vez que afetam os valores dos coeficientes de regressão estimados (FIELD, 2009). Ainda de acordo com Field (2009), a identificação de outliers deve ser realizada através da análise de distância de Mahalanobis, que medem os afastamentos dos valores a partir das médias das variáveis preditoras.

Para o seguinte trabalho, foram considerados 35 graus de liberdade, que correspondem ao número de itens da variável independente, valores organizacionais, e com o índice de significância estatística $\mathrm{p}<0,001$, obteve-se, segundo consulta à Tabela Qui-Quadrado, o valor de $X^{2}=66,619$. A partir desse valor, foram encontrados três outliers que foram excluídos da amostra. Destarte, foram considerados 97 sujeitos, atingindo a amostra mínima necessária, com poder estatístico de $80 \%$, de acordo com o software GPower.

Para a regressão múltipla linear, seguiu-se as suposições de regressão propostas por Hairet al. (2009), realizando análises por meio de gráficos de probabilidade normal e gráficos de resíduos a fim de verificar a linearidade do fenômeno, a homocedasticidade (variância constante dos termos de erro) e a normalidade da distribuição dos termos de erro.

Ainda, foram realizadas as análises de multicolinearidade, que não apresentaram problemas para a amostra pesquisada, uma vez que os valores de tolerance (tolerância) foram superiores a 0,1 e os valores referentes avarianceinflationfactor (fator de inflação da variância - VIF) foram inferiores a 10 (HAIR et al., 2009).

Para estudar a relação entre as variáveis de estudo, foi utilizado um modelo de pesquisa que adota como variável independente os Valores Organizacionais e como variável dependente as Políticas e Práticas de GP.

O instrumento utilizado para abordar a variável políticas e práticas de gestão de pessoas foi a Escala de Políticas e Práticas de Recursos Humanos (EPPRH), validada no Brasil e também nos EUA por Demo e Rozzett (2012), apresentando um modelo atual, abrangente e com melhores índices de ajuste. Originalmente com 32 itens distribuídos em 6 fatores. No entanto, foram retirados dois itens do fator recrutamento e seleção por não 
representarem a realidade da empresa pesquisada, uma vez que a mesma é uma empresa pública. Os fatores possuem índices de confiabilidade entre 0,81 e 0,91.

A segunda escala é referente à variável de valores organizacionais, validado por Porto et al. (2013). Foi escolhida por ser a escala mais atual validada e adaptada à realidade brasileira. O instrumento é composto por 38 itens, distribuídos em 6 fatores, baseados em seis tipos motivacionais: inovação, estabilidade, hierarquia, igualitarismo, domínio e harmonia. Os fatores possuem índices de confiabilidade variando entre 0,72 e 0,91 . Para adequação do instrumento à realidade da organização estudada, foram retirados três itens que não faziam jus à empresa pesquisada. As variáveis de estudo encontram-se definidas no Quadro 1 .

Quadro 1- Variáveis de estudo

\begin{tabular}{|c|c|c|c|}
\hline Variável & Classificação & Fatores & Descrição \\
\hline \multirow{6}{*}{$\begin{array}{c}\text { Valores } \\
\text { Organizacionais }\end{array}$} & \multirow{6}{*}{ Independente } & Hierarquia & $\begin{array}{c}\text { Legitimidade da distribuição desigual do } \\
\text { poder, papéis e recursos (Porto et al., } \\
\text { 2013). }\end{array}$ \\
\hline & & Igualitarismo & $\begin{array}{l}\text { Prioridade dos interesses do grupo e bem- } \\
\text { estar dos demais em relação ao interesses } \\
\text { pessoais (Porto et al., 2013). }\end{array}$ \\
\hline & & Domínio & $\begin{array}{c}\text { Posicionamento que objetiva dominar e } \\
\text { mudar o mundo, numa tentativa de } \\
\text { demonstração de controle (Porto et al., } \\
\text { 2013). }\end{array}$ \\
\hline & & Harmonia & $\begin{array}{l}\text { Adequar-se ao mundo ao invés de mudá- } \\
\text { lo, ajustar-se de maneira harmoniosa com } \\
\text { o ambiente (Porto et al., 2013). }\end{array}$ \\
\hline & & Inovação & $\begin{array}{l}\text { Capacidade de a organização buscar } \\
\text { novidades e inovar (Porto et al., 2013). }\end{array}$ \\
\hline & & Estabilidade & $\begin{array}{l}\text { Manutenção do status quo (Porto et al., } \\
\text { 2013). }\end{array}$ \\
\hline \multirow{2}{*}{$\begin{array}{l}\text { Políticas e Práticas } \\
\text { de Gestão de } \\
\text { Pessoas }\end{array}$} & \multirow[t]{2}{*}{ Dependente } & $\begin{array}{l}\text { Recrutamento e } \\
\text { Seleção }\end{array}$ & $\begin{array}{l}\text { Proposta articulada da organização, com } \\
\text { construções teóricas e práticas, para } \\
\text { procurar colaboradores, estimulá-los a se } \\
\text { candidatar e selecioná-los, buscando } \\
\text { harmonizar os valores, interesses, } \\
\text { expectativas e competências da pessoa } \\
\text { com as características e demandas do } \\
\text { cargo e da organização (DEMO et al } \\
\text { 2011). }\end{array}$ \\
\hline & & Envolvimento & $\begin{array}{l}\text { Proposta articulada da organização com } \\
\text { construções teóricas e práticas para criar } \\
\text { um vínculo afetivo com seus } \\
\text { colaboradores, contribuindo para seu bem- } \\
\text { estar, em termos de reconhecimento, } \\
\text { relacionamento, participação } \\
\text { comunicação (DEMO et al 2011). }\end{array}$ \\
\hline
\end{tabular}




\begin{tabular}{|c|c|c|c|}
\hline Variável & Classificação & Fatores & Descrição \\
\hline & & $\begin{array}{l}\text { Treinamento, } \\
\text { Desenvolvimento } \\
\text { e Educação }\end{array}$ & $\begin{array}{l}\text { Proposta articulada da organização, com } \\
\text { construções teóricas e práticas, para } \\
\text { provar aos colaboradores a aquisição } \\
\text { sistemática de competências e estimular a } \\
\text { contínua aprendizagem e produção de } \\
\text { conhecimento (DEMO et al 2011). }\end{array}$ \\
\hline & & $\begin{array}{l}\text { Condições de } \\
\text { Trabalho }\end{array}$ & $\begin{array}{l}\text { Proposta articulada da organização, com } \\
\text { construções teóricas e práticas, para } \\
\text { prover aos colaboradores boas condições } \\
\text { de trabalho em termos de benefícios, } \\
\text { saúde, segurança e tecnologia (DEMO et } \\
\text { al 2011). }\end{array}$ \\
\hline & & $\begin{array}{l}\text { Avaliação de } \\
\text { Desempenho e } \\
\text { Competências }\end{array}$ & $\begin{array}{l}\text { Proposta articulada da organização, com } \\
\text { construções teóricas e práticas, para } \\
\text { avaliar o desempenho e as competências } \\
\text { dos colaboradores, subsidiando as } \\
\text { decisões sobre promoções, planejamento } \\
\text { de carreira e desenvolvimento (DEMO et } \\
\text { al 2011). }\end{array}$ \\
\hline & & $\begin{array}{l}\text { Remuneração } \\
\text { Recompensas }\end{array}$ & $\begin{array}{l}\text { Proposta articulada da organização, com } \\
\text { construções teóricas e práticas, para } \\
\text { recompensar o desempenho e as } \\
\text { competências dos colaboradores em } \\
\text { termos de remuneração e incentivos } \\
\text { (DEMO et al 2011). }\end{array}$ \\
\hline
\end{tabular}

Fonte:Adaptado de Demo et al. (2011) e Porto et al. (2013)

Em relação à análise de dados, para a identificação das variáveis foi realizada uma análise descritiva simples, abrangendo o cálculo das médias e desvios-padrão das respostas. Para a análise da relação entre as variáveis foi utilizado o método de regressão linear múltipla que, segundo Tabachink e Fidell (2013), possibilita a avaliação do relacionamento de uma variável dependente com diversas variáveis independentes, obtendo como resultado uma equação linear de predição da variável dependente.

O escalonamento multidimensional (Multidimensional Scaling - MDS) é uma técnica exploratória multivariada que permite representar as proximidadades entre um conjunto de objetos, com base em um conjunto de atributos multivariados medidos (Marôco, 2011). Essa técnica é semelhante à análise fatorial, e foi utilizada para identificar as dimensões da escala de valores organizacionais.

Foi utilizado, no presente estudo, o MDS clássico, cujos dados consistem em uma matriz de proximidades (semelhanças/dessemelhanças) entre os objetos (Marôco, 2011). Para avaliar a qualidade da solução, alguns recursos podem ser utilizados por meio de uma ou mais funções de discrepância (STRESS), quanto maior o STRESS-I pior é a qualidade do modelo 
(Marôco, 2011). O cálculo da Dispersão das Proximidades também é utilizado para avaliar a qualidade, e é explicado pela solução DAF (DispersionAccounted For), quanto mais próximo de 1, melhor será a solução (Marôco, 2011). Esses dados foram medidos no modelo utilizado e todos foram considerados satisfatórios.

A Figura 1 revela a estrutura apresentada no MDS. Itens que se localizaram em região oposta à previsão teórica foram eliminados. Do total de 35 itens, a análise apontou para a adequação de 23 itens.

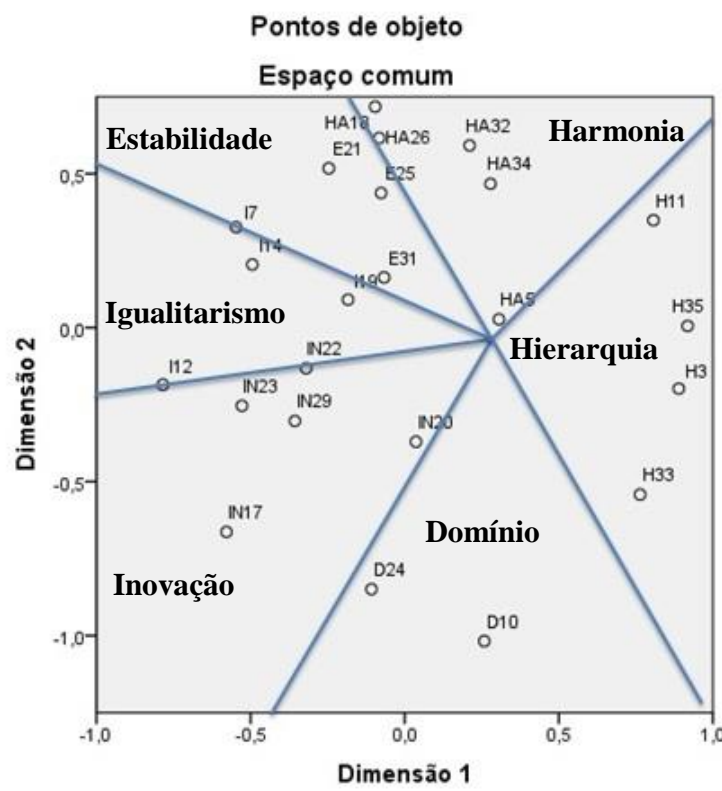

Figura 1: Escalonamento Multidimensional Fonte:Elaborado pelas autoras

\section{RESULTADOS E DISCUSSÕES}

Para que o objetivo da pesquisa fosse alcançado - avaliar se os valores organizacionais são preditores das políticas e práticas de GP na organização estudada - foram avaliadas as correlações entre as variáveis do modelo de pesquisa. A Tabela 1 sintetiza esses resultados.

Tabela 1 - Correlação dos fatores 


\begin{tabular}{|c|c|c|c|c|c|c|c|c|c|c|c|c|c|}
\hline & & HIERARQ & HARM & DOM & IGUAL & INOV & ESTAB & RS & ENV & TDE & COND & AVAL & RECOMP \\
\hline \multirow{6}{*}{ VO } & HIERARQ & 1 & & & & & & & & & & & \\
\hline & HARM & ,223* & 1 & & & & & & & & & & \\
\hline & $\mathrm{DOM}$ & 0,115 & ,261" & 1 & & & & & & & & & \\
\hline & IGUAL & 0,012 & ,706" & ,281* & 1 & & & & & & & & \\
\hline & INOV & $-0,021$ & ,591" & , 436* & , $835^{* *}$ & 1 & & & & & & & \\
\hline & ESTAB & 0,157 &, $748^{* *}$ &, $303^{* *}$ & ,731" & ,623** & 1 & & & & & & \\
\hline \multirow{6}{*}{ PPGP } & RS & 0,177 & 287" & $-0,055$ & 0,19 & 0,187 & ,220* & 1 & & & & & \\
\hline & ENV & $-0,087$ & ,438** &, $255^{*}$ & ,704** & ,647" & ,488 & ,432** & 1 & & & & \\
\hline & TDE & 0,123 & , $336 *$ & 0,146 &, $545^{* *}$ &, 556 & $344^{* *}$ &, $371^{*}$ &, $704^{* *}$ & 1 & & & \\
\hline & COND & $-0,002$ & ,264 & 0,145 &, $511^{* *}$ & ,428** &, $443^{* *}$ & ,206* & ,537 & , 418** & 1 & & \\
\hline & AVAL & 0,071 & ,381" & 0,166 & , 409** & $388^{* *}$ & ,248* & ,391* & , 476 & , $448^{* *}$ &, $413^{* *}$ & 1 & \\
\hline & RECOMP & ,208* & ,394* &, $208^{*}$ & ,428** & ,389" &, $318^{* *}$ &, $363^{* *}$ & ,614 &, $571^{* *}$ & ,421" & ,670 & 1 \\
\hline
\end{tabular}

*.A correlação é significativa a nível 0,05 (2 extremidades).

**.A correlação é significativa a nível 0,01 (2 extremidades).

Fonte: Elaborado pelas autoras

Nota-se que a grande maioria foi significativa e positiva no nível 0,01. De acordo com Cohen (1992), valores entre 0,1 e 0,29 apresentam baixa associação; entre 0,3 e 0,49 apresentam média associação e maiores que 0,5 apresentam forte associação. As correlações significativas mais fortes encontradas foram envolvimento e igualitarismo $(0,704)$, envolvimento e inovação $(0,647)$, TD\&E e igualitarismo $(0,545)$, TD\&E e inovação $(0,556)$, condições de trabalho e igualitarismo $(0,511)$. Considerando que a maioria das correlações entre as variáveis foram significativas, recomenda-se estimar relações de predição entre as variáveis através de regressões lineares (FIELD, 2009).

Foram realizadas seis regressões múltiplas lineares, uma vez que o método de regressão múltipla linear identifica os melhores preditores, segundo o conceito de Tabachnick e Fidell (2013), levando em consideração os seis valores organizacionais como variável independente e as seis políticas e práticas de GP como variável dependente.

Para os fatores recrutamento e seleção, avaliação de desempenho e competências e remuneração e recompensas nenhum valor organizacional foi preditor, uma vez que os fatores da variável independente não foram significativos $(\mathrm{p}>0,05)$.

Em relação ao fator envolvimento, o $\mathrm{R}^{2}$ foi igual a 0,517 , ou seja, o envolvimento pode ser explicado em 51,7\% pelo valor igualitarismo, que apresentou $\beta=0,609$, obtendo uma relação positiva e um grande efeito sobre o envolvimento. Ou seja, quanto mais os servidores percebem o valor de igualitarismo como presente na organização, mais percebem práticas de envolvimento, quais sejam, comunicação, participação, reconhecimento e relacionamento. 
Tabela 2 - Parâmetros da regressão linear para o fator Envolvimento

\begin{tabular}{c|c|c|c|c|c|c}
\hline VD & VI & $\mathbf{R}^{2}$ & Variáveis & B & Sig & $\begin{array}{c}\text { Significância } \\
\text { estatística }\end{array}$ \\
\hline \multirow{2}{*}{ Envolvimento } & $\begin{array}{c}\text { Valores } \\
\text { Organizacionais }\end{array}$ & $\begin{array}{c}0,517 \\
\text { ou } \\
51,7 \%\end{array}$ & Igualitarismo & 0,609 & 0,000 & Sig=0,000 \\
\hline
\end{tabular}

Fonte: Elaborado pelas autoras

De acordo com Mendes e Tamayo (2001), a estrutura igualitária está relacionada com o prazer e a valorização no trabalho, isso quer dizer que o sentimento de valorização também ocorre quando os colaboradores percebem um enfoque organizacional em aspectos como democracia, descentralização, justiça, sociabilidade, coleguismo, igualdade, cooperação, entre outros.

Outrossim, a política de envolvimento é uma proposta organizacional que objetiva a criação de um vínculo afetivo entre seus funcionários, contribuindo para seu bem-estar, em termos de reconhecimento, relacionamento, participação e comunicação (DEMO et al., 2011).Uma vez que o igualitarismo se relaciona com prazer e sentimento de valorização, e a política de envolvimento se relaciona com o bem-estar no ambiente de trabalho, com valores de igualitarismos presentes no ambiente organizacional por meio de práticas de comunicação e participação, os funcionários estarão mais suscetíveis a se envolverem com a organização.

Em relação ao fator TD\&E, o $\mathrm{R}^{2}$ foi igual a 0,371 , ou seja, as práticas de treinamento, desenvolvimento e educação podem ser explicadas em $37,1 \%$ pelos valores de igualitarismo e inovação, que apresentaram respectivamente $\beta=0,387$ e $\beta=0,416$, obtendo uma relação positiva e um grande efeito. Em outras palavras, quanto mais os servidores percebem os valores de igualitarismo e inovação, mais percebem as práticas de TD\&E da organização. O valor referente à Inovação é considerado o melhor preditor uma vez que possui um $\beta$ maior $(\beta=0,416)$.

Tabela 3 - Parâmetros da regressão linear para o fator TD\&E

\begin{tabular}{c|c|c|c|c|c|c}
\hline VD & VI & $\mathbf{R}^{2}$ & Variáveis & B & Sig & $\begin{array}{c}\text { Significância } \\
\text { estatística }\end{array}$ \\
\hline \multirow{2}{*}{ TD\&E } & $\begin{array}{c}\text { Valores } \\
\text { Organizacionais }\end{array}$ & $\begin{array}{c}0,371 \\
\text { ou } \\
37,1 \%\end{array}$ & Igualitarismo & 0,387 & 0,033 & $\mathrm{~F}=8,861$ \\
& & & & & & \\
\end{tabular}


Fonte: Elaborado pelas autoras

A dimensão de inovação está relacionada com o estimulo à criatividade, a curiosidade dos funcionários e a criação e implementação de novos produtos, serviços ou processos, ou seja, a organização sempre está em busca de mudanças (PORTO et al., 2013).

As práticas de TD\&E buscam prover aos funcionários uma aquisição sistemática de competências e estimular a aprendizagem e a produção de conhecimento (DEMO et al., 2011). Dessa forma, essas práticas se tornam cada vez mais importantes para o sucesso das organizações, pois são essenciais para a implementação de estratégias inovadoras, permitindo o desenvolvimento e a consolidação das competências necessárias para o alcance os objetivos organizacionais (BOHLANDER, SNELL; 2009). Com isso, nota-se que as organizações precisam de uma base desenvolvedora, a partir de práticas de TD\&E, para que os valores relacionados à inovação possam ser colocados em prática e serem bem sucedidos.

O igualitarismo é definido por Porto e Tamayo (2005) como o bem-estar organizacional acima dos interesses individuais. Como as práticas de TD\&E visam o desenvolvimento dos servidores, impactam também o bem-estar do ambiente organizacional e dos próprios servidores.

Com relação ao último fator, condições de trabalho, o $\mathrm{R}^{2}$ foi igual a 0,315 , o que significa dizer que $31,5 \%$ de condições de trabalho podem ser explicadas pelos valores de igualitarismo e harmonia, o que significa dizer que quanto mais os valores de igualitarismo e harmonia são percebidos, mas os servidores notarão as práticas de condições de trabalho. As relações são positivas entre os fatores dependentes e independentes, além de possuírem um grande efeito.Como igualitarismo possui um $\beta$ maior, é considerado o melhor preditor.

Tabela 4 - Parâmetros da regressão linear para o fator Condições de Trabalho

\begin{tabular}{c|c|c|c|c|c|c}
\hline VD & VI & $\mathbf{R}^{2}$ & Variáveis & B & Sig & $\begin{array}{c}\text { Significância } \\
\text { estatística }\end{array}$ \\
\hline \multicolumn{1}{c}{$\begin{array}{c}\text { Condições de } \\
\text { Trabalho }\end{array}$} & Valores & $\begin{array}{c}0,315 \\
\text { Ou }\end{array}$ & Igualitarismo & 0,527 & 0,006 & $\mathrm{~F}=4,349$ \\
& Organizacionais & $31,5 \%$ & Harmonia & 0,314 & 0,032 & Sig=0,01
\end{tabular}

Fonte: Elaborado pelas autoras

Os valores relativos ao igualitarismo referem-se à autotranscendência dos interesses pessoais em favor do bem-estar dos demais (PORTO et al, 2013). As condições de trabalho 
estão relacionadas às condições físicas de trabalho, bem-estar e saúde, segurança e benefícios, que devem estar baseados nas melhores práticas organizacionais (SINGAR, RAMSDEN; 1972).

Dessa forma, os valores de igualitarismo possuem interface com o suporte organizacional (TAMAYO, MENDES, PAZ; 2000), uma vez que o fator condições de trabalho está relacionado a benefícios, saúde, segurança e tecnologia (DEMO et al., 2011).Quanto mais a organização se mostrar igualitária - aumentando o suporte prestado aos colaboradores - mais os mesmos perceberão positivamente práticas de condições de trabalho.

Os valores associados à harmonia, segundo Tamayo, Mendes e Paz (2000), referem-se aos valores que enfatizam a harmonia com o ambiente e com outras organizações. As organizações que valorizam a harmonia, buscam se relacionar com o ambiente externo por meio da colaboração, e não exploração (PORTO, TAMAYO; 2005). Essa interação harmoniosa entre a empresa e o ambiente, pode impactar também a interação entre os servidores, que perceberão o ambiente de trabalho de maneira mais positiva, impactando na percepção das condições de trabalho, até porque harmonia também se experimenta em um bom ambiente físico, ergonômico e emocional de trabalho.

Por fim, comparou-se os resultados por ora obtidos com os de outros estudos de tônica similar. Os resultados encontrados no estudo de Carvalho, Oliveira e Silva (2013) relataram que para os servidores pesquisados, os valores mais relevantes são os de autonomia, hierarquia e conservadorismo. Apesar do presente estudo também ter foco no serviço público, os resultados divergiram. O único valor compartilhado com o estudo de Carvalho, Oliveira e Silva (2013) é o de autonomia (nesta pesquisa, tratado como inovação).

Os resultados do estudo de Demo (2010) mostraram que os valores pessoais de poder e universalismo impactam as percepções de funcionários de empresas publicas e privadas sobre políticas e práticas de GP. Como os valores pessoais e organizacionais possuem a mesma origem teórica (Schwartz, 1999), a comparação entre os resultados pode ser feita. Para o presente estudo, o valor pessoal de universalismo - nas organizações sendo identificado como harmonia - também foi encontrado como relevante preditor da política de envolvimento, tal qual apontado por Demo (2010).

A Figura 2 sumariza o resumo dos resultados encontrados nesta pesquisa, desvelando os valores organizacionais preditores de cada política de GP. 


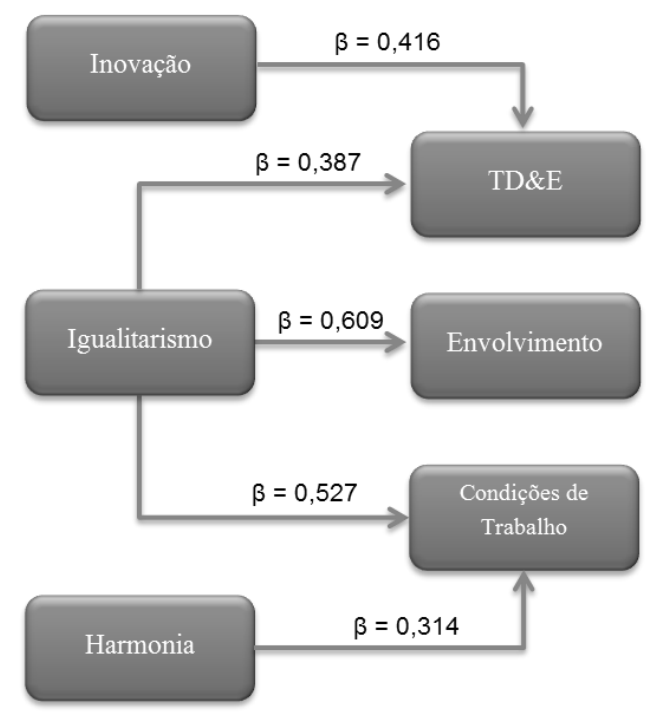

Figura 2 - Esquema dos resultados das análises de regressão

Fonte: Elaborado pelas autoras

\section{CONSIDERAÇÕES FINAIS}

O principal objetivo deste estudo foi identificar as relações existentes entre valores organizacionais e políticas e práticas de gestão de pessoas na realidade de uma empresa pública do Distrito Federal. O resultado apresentou que os valores relativos à igualitarismo, predizem três políticas e práticas de GP: condições de trabalho, TD\&E e envolvimento. Além disso, os valores concernentes à inovação predizem as políticas e práticas de TD\&E; e os valores concernentes à harmonia predizem as práticas de condições de trabalho.

O estudo desenvolvido contribuiu para a literatura acadêmica de Gestão de Pessoas e de Comportamento Organizacional, principalmente considerando a inexistência de outros estudos que analisem a relação entre as duas variáveis, utilizando as escalas mais novas de políticas e práticas de GP e valores organizacionais. Também contribuiu com uma lacuna na literatura a respeito de políticas e práticas de GP como variável dependente, uma vez que grande parte dos estudos sobre o tema utiliza a variável como independente (DEMO, 2010; RUBINO, DEMO, TRALDI, 2011; HORTA, DEMO, ROURE, 2012; DEMO, MARTINS, ROURE, 2012).

A revisão da produção nacional e a institucionalização de pesquisa das suas variáveis produzidas neste trabalho contribuíram para identificação de lacunas sobre os estudos das variáveis, permitindo a elaboração de uma agenda de pesquisa. 
Além disso, a contribuição prática do estudo está baseada no diagnóstico realizado, os valores organizacionais predizendo as políticas e práticas de GP, e que podem auxiliar os gestores da organização estudada a identificar os valores organizacionais percebidos e o impacto dos mesmos nas rotinas organizacionais, informações que podem contribuir para futuras tomadas de decisões.

Quanto às limitações, por se tratar de um estudo de corte transversal seus resultados são limitados ao momento de aplicação da pesquisa e à amostra considerada por ela. Esse caráter transversal aliado à técnica estatística utilizada também não permitem traçar nenhuma inferência sobre causalidade entre as variáveis, apenas relações de predições foram estimadas.

Considerando as limitações apontadas e as lacunas na literatura identificadas por meio da revisão da produção nacional realizada, é possível a proposição de uma agenda para estudos futuros. No que se refere às políticas e práticas de GP, observa-se uma carência de medidas, o que pode ser traduzido em um apelo a mais esforços e pesquisas que validem instrumentos diagnósticos. Outra lacuna que pode ser observada é a baixa produção de ensaios teóricos e estudos qualitativos e multimétodo sobre as duas variáveis, que estudam a variável de maneira mais profunda, discutindo conceitos e fundamentos e realizando revisões bibliográficas e bibliométricas que privilegiam a compreensão da variável em estudo. Por fim, sugerem-se mais estudos com enfoque na área pública, uma vez que a maior parte dos estudos investiga a área privada.

No que tange à variável de valores organizacionais, também foi detectada uma lacuna na produção de estudos sobre medidas, inclusive a escala por ora utilizada ainda está no prelo, mas já foi utilizada em outros estudos (NASCIMENTO, 2014; SOUSA, 2013) evidenciando os esforços da academia em produzir melhores instrumentos de valores.

É válido ressaltar a participação da Universidade de Brasília na produção de artigos sobre as duas variáveis de estudo. A institucionalização de pesquisa permitiu identificar que a universidade possui grupos de pesquisa consolidados com representatividade nos dois temas. Além disso, o levantamento de autores que mais publicaram sobre as variáveis na revisão da produção nacional realizada, apresenta representantes da Universidade de Brasília para ambos os temas.

Por fim, os resultados e recomendações deste estudo não são conclusivos ou limitantes, porém, representam um esforço inicial de investigação da relação entre as variáveis consideradas. É importante ressaltar a necessidade de constante atualização e 
realização de pesquisas para aprofundar o conhecimento sobre esta relação, com o objetivo de contribuir para o avanço relativo às políticas e práticas de GP e os valores organizacionais, elementos que constituem o núcleo da cultura organizacional.

\section{REFERÊNCIAS}

AGUZOLLI, R.; GEARY, J. An 'emerging challenge': The employment practices of a Brazilian multinational company in Canada. Human Relations, Thousand Oaks, v. 67, n. 5, p. 587-609, 2014.

ALDAMOE, F. M. A.; YAZAM, M.; AHMID, K. B. The mediating effect of HRM outcomes (employee retention) on the relationship between HRM practices and organizational performance. International Journal of Human Resource Studies, Las Vegas, v. 2, n. 1, p. 75-88, 2011.

ARMSTRONG, M. Armstrong's handbook of human resource management practice. 12. ed. London: Kogan Page, 2012.

BARDIN, L. Análise de Conteúdo. Lisboa: Edições 70, 2011.

BARNEY, J. Firm resources and sustained competitive advantage. Journal of Management, Thousand Oaks, v. 17, n. 1, p. 99-120, 1991.

BOHLANDER, G. W.; SNELL, S. Administração de recursos humanos. 14. ed. São Paulo: Cengage Learning. 2009.

BOSELIE, P.; DIETZ, G.; BOONE, C. Comunalities and contradictions in HRM and performance research. Human Resource Management Journal, Hoboken, v. 15, n. 3, p. 6794, 2005.

CANTARELLO, S.; FILIPPINI, R.; NOSELLA, A. Linking human resources management practices and customer satisfaction on product quality. The International Journal of Human Resources Management, Abingdon, v. 13, n. 18, p. 3906-3924, 2012.

CARVALHO, V. D.; OLIVEIRA, T. A.; SILVA, D. C. Valores organizacionais em instituições públicas brasileiras: percepções dos servidores em diferentes posições hierárquicas e tipos de entidade de administração indireta. RAM - Revista de Administração Mackenzie, São Paulo, v. 14, n. 5, p. 74-103, set./out. 2013.

COHEN, J. A power primer. Psychological Bulletin, New York, v. 112, n. 1, p. 155-159, 1992.

COMBS, J. et al. How much do high-performance work practices matter? A meta-analysis of their effects on organizational performance. Personnel Psychology, Hoboken, v. 59, n. 3, p. 501-528, 2006. 
DEMO, G. Políticas de gestão de pessoas nas organizações: estado da arte, produção nacional, agenda de pesquisa, medidas e estudos relacionais. São Paulo: Atlas, 2012.

DEMO, G. Políticas de Gestão de Pessoas, valores pessoais e justiça organizacional. RAM Revista de Administração Mackenzie, São Paulo, v. 11, n. 5, set./out. 2010.

DEMO, G. et al. Políticas de gestão de pessoas no novo milênio: cenário dos estudos publicados nos periódicos da área de administração entre 2000 e 2010. Revista de Administração Mackenzie, São Paulo, v. 12, n. 5, p. 15-42, 2011.

DEMO, G. et al. Políticas e práticas de recursos humanos. In: SIQUEIRA, M. M. (Org.). Novas medidas do comportamento organizacional: ferramentas de diagnóstico e de gestão. Porto Alegre: Artmed, 2014. p. 240-255.

DEMO, G; ROZZETT, K. Human Resource Management Policies and Practices Scale (HRMPP): scale validation in the United States. International Journal of Strategic Management, Stockton, v. 12, n. 3, p. 41-66, 2012.

DESSLER, G. Human resource management. 9. ed. New Jersey: Prentice Hall, 2002.

EZZAMEL, M.; LILLEY, S.; WILLMOTT, H. Practices and practicalities in human resource management. Human Resource Management Journal, Hoboken, v. 6, n. 1, p. 63-80, 1996.

FIELD, A. Discovering statistics using SPSS. 3. Ed. London: Sage, 2009.

FRENKEL, S.; RESTUBOG, S. L.; BEDNALL, T. How employee perceptions of HR policy and practice influence discretionary work effort and co-worker assistance: evidence from two organizations. The International Journal of Human Resource Management, Abingdon, v. 23, n. 20, p. 4193-4210, 2012.

GELADE, G. A.; IVERY, M. The impact of human resource management and work climate on organizational performance. Personnel Psychology, Hoboken, v. 56, p. 383-404, 2003.

GOMIDE JR., S.; TANABE, T. R. Políticas de gestão de pessoas e efetividade organizacional. In: DEMO, G. (Org.). Políticas de gestão de pessoas nas organizações: estado da arte, produção nacional, agenda de pesquisa, medidas e estudos relacionais. São Paulo: Atlas, 2012. p. 175-195.

GOULD-WILLIAMS, J. The importance of HR practices and workplace trust in achieving superior performance: a study of public-sector organizations. International Journal of Human Resource Management, Abingdon, v. 14, n. 1, p. 28-54, 2003.

GUARDANI, F. et al. A relação entre valores, práticas organizacionais e confiança de clientes no setor de serviços. Produção, Florianópolis, v. 23, n. 4, p. 806-817, out./dez. 2013.

GUEST, D. Human resource management and industrial relations. The Journal of Management Studies, Hoboken, v. 24, n. 5, p. 503-521, 1987. 
GUEST, D.; CONWAY, N. The impact of HR practices, HR effectiveness and a 'strong HR system' on organizational outcomes: a stakeholder perspective. The International Journal of Human Resource Management, Abingdon, v. 22, n. 8, p. 1686-1702, 2011.

GUEST, D.; HOQUE, K. Yes, personnel does make a difference. Personnel Management, New York, v. 26, n. 11, p. 40-43, 1994.

HAIR, J. F. et al. Análise multivariada de dados. 6. ed. Porto Alegre: Bookman, 2009.

HASSAN, A. Human resource development and organizational values. Journal of European Industrial Training, Bingley, v. 31, n. 6, p. 435-448, 2007.

HOFSTEDE, G. et al. Measuring organizacional cultures: A qualitative and quantitative study across twenty cases. Administrative Science Quarterly, Thousand Oaks, v. 35, n. 2, p. 286316, 1990.

HORTA, P.; DEMO, G.; ROURE, P. Políticas de gestão de pessoas, confiança e bem-estar: estudo em uma multinacional. Revista de Administração Contemporânea, Rio de Janeiro, v. 16, n. 4, p. 566-585, jul./ago. 2012.

HUSELID, M. A.; JACKSON, S. E.; SCHULER, R. S. Technical and strategic human resource management effectiveness as a determinant of firm performance. Academy of Management Journal, Briarcliff Manor, v. 40, n. 1, p. 171-188, 1997.

INYANG, B. J.; AKAEGBU, J. B. Redefining the Role of the Human Resource Professional (HRP) in the Nigerian Public Service for Enhanced Performance. International Journal of Business Administration, Toronto, v. 5, n. 1, p. 90-98, 2014.

JIA, L. et al. A social-structural perspective on employee-organization relationships and team creativity. Academy of Management Journal, Briarcliff Manor, v. 57, n. 3, p. 869-891, 2014.

KATOU, A. A. Investigating reverse causality between human resource management policies and organizational performance in small firms. Management Research Review, Bingley, v. 35, n. 2, p. 134-156, 2012.

KIM, A.; LEE, C. How does HRM enhance strategic capabilities? Evidence from the Korean management consulting industry. The International Journal of Human Resource Management, Abingdon, v. 23, n. 1, p. 126-146, 2012.

LEGGE, K. Human resource management. In: Ackroyd, S. et al. (Eds.). The Oxford handbook of work and organization. Oxford: Oxford University Press, 2006.

LEGGE, K. Human resource management: rethorics and realities. London: Macmillan, 1995. 
MAJUMDER, M. T. H. HRM practices and employees'satisfaction towards private banking sector in Bangladesh. International Review of Management and Marketing, Yenice, v. 2, n. 1, p. 52-58, 2012.

MARIOTTI, D. F.; SOUZA, Y. S. Relações de confiança na dinâmica de uma organização. In: ENCONTRO NACIONALDA ASSOCIAÇÃO NACIONALDE PÓS-GRADUAÇÃ̃OE PESQUISAEM ADMINISTRAÇÃO, 29., 2009, Brasília. Anais... Brasília: ANPAD, 2009.

MARÔCO, J. Análise estatística com o SPSS Statistics. 5. Ed. Pero Pinheiro: Report Number, 2011.

MARTÍN-ALCÁZAR, F.; ROMERO-FERNÁNDEZ, P. M.; SÁNCHEZ-GARDEY, G. Strategic human resource management: integrating the universalistic, contingent, configurational and contextual perspectives. International Journal of Human Resource Management, Abingdon, v. 16, n. 5, p. 633-659, 2005.

MASCARENHAS, A. O. Gestão estratégica de pessoas: evolução, teoria e crítica. São Paulo: Cengage Learning, 2009.

MASCARENHAS, A. O.; KIRSCHBAUM, C. Fundamentos da gestão estratégica de pessoas. In: MASCARENHAS, A. O. (Org.). Gestão estratégica de pessoas: evolução, teoria e crítica. São Paulo: Cengage Learning, 2008. p. 23-30.

MATHIS, R. L.; JACKSON, J. H. Human resource management. 10. ed. Ohio: SouthWestern/Thomson. 2003.

MENDES, A. M.; TAMAYO, A. Valores organizacionais e prazer-sofrimento no trabalho. Psico-USF, São Paulo, v. 6, n. 1, p. 39-46, jan./jun. 2001.

MENEZES, L. M.; WOOD, S.; GELADE, G. The integration of human resource and operation management practices and its link with performance: a longitudinal latent class study. Journal of Operations Management, Amsterdam, v. 28, p. 455-471, 2010.

MORRIS, S.; SNELL, S. The Evolution of HR Strategy: Adaptations to Increasing Global Complexity. In: WILKINSON, A. et al. (Eds.). The SAGE handbook of human resource management. London: Sage, 2010. p. 84-99.

NASCIMENTO, T. G. Desempenho competente: relações com valores, práticas e identidade no serviço policial. 2014. Tese (Doutorado) - Departamento de Administração, Universidade de Brasília, Brasília, 2014.

NISHII, L. H.; LEPAK, D. P.; SCHNEIDER, B. Employee attributions of the "Why" of HR practices: their effects on employee attitudes and behaviors, and customer satisfaction.

Personnel Psychology, Hoboken, v. 61, p. 503-545, 2008.

OLIVEIRA, A. F.; TAMAYO, A. Inventário de Perfis de Valores Organizacionais. Revista de Administração da Universidade de São Paulo, São Paulo, v. 39, n. 2, p, 129-149, abr./jun. 2004. 
OLIVEIRA, A. M. B.; OLIVEIRA, A. J.; Gestão de Recursos Humanos: uma Metanálise de seus Efeitos sobre o Desempenho Organizacional. Revista de Administração Contemporânea, Curitiba, v. 15, n. 4, p. 650-669, jul./ago. 2011.

OSBORN, R.; HUNT, J.; SCHERMERHORN, J. Fundamentos de comportamento organizacional. 2. ed. São Paulo: Bookman, 1998.

PORTO, J. B. et al. Valores Organizacionais: Evidências de adequação da Teoria de Valores Culturais de Schwartz. Manuscrito submetido para publicação. 2013.

PORTO, J. B.; TAMAYO, A. Valores organizacionais e civismo nas organizações. RAC Revista de Administração Contemporânea, Curitiba, v. 9, n. 1, p. 35-52. 2005.

RUBINO, T.; DEMO, G.; TRALDI, M. T. F. As políticas de gestão de pessoas influenciam o bem-estar no trabalho? CONGRESSO IBEROAMERICANO DE PSICOLOGIADAS ORGANIZAÇÕESEDO TRABALHO, 2., 2001, Florianópolis. Anais... Florianópolis: CFP, 2011.

SANTOS, M. J. N. Gestão de recursos humanos: teorias e práticas. Sociologias, Porto Alegre, v. 6, n. 12, p. 142-158, jul./dez. 2004.

SCHNEIDER, B.; BOWEN, D. Employee and customer perceptions of service in banks: Replication and extension. Journal of Applied Psychology, Washington, v. 70, n. 3, p. 423433, 1985.

SCHWARTZ, S. H. A theory of cultural values and some implications for work. Applied Psychology-an International Review, Hoboken, v. 48, n. 1, p. 23-47. 1999.

SERPELL, A.; FERRADA, X. A competency-based model for construction supervisors in developing countries. Personnel Review, Bingley, v. 36, n. 4, p. 585-602. 2007.

SINGAR, E. J.; RAMDSEN, J. Human Resources: obtaining results from people at work. New York: McGraw-Hill, 1972.

SOUSA, J. M. 2013. Felicidade no trabalho: impacto de valores laborais e organizacionais. Dissertação não publicada (Mestrado) - Instituto de Psicologia, Universidade de Brasília, Brasília, 2013.

STONE, D. L.; STONE-ROMERO, E. F.; LUKASZEWSKI, K. The impact of cultural values on acceptance and effectiveness of human resource management policies and practices.

Human Resource Management Review, Amsterdam, v. 17, p. 152-165, 2007.

STOREY, J. New perspectives in human resource management. London: Routledge, 1995.

SUBRAMONY, M. A meta-analytic investigation of the relationship between HRM bundles and firm performance. Human Resource Management, Hoboken, v. 48, n. 5, p. 745-768, 2009. 
TABACHNICK, B.; FIDELL, L. S. Using multivariate statistics. 5. Ed. Boston: Allyn and Bacon, 2013.

TAMAYO, A; BORGES, L. O. Valores do trabalho e das organizações. In: ROS, M.; GOUVEIA, V. V. (Orgs.). Psicologia social dos valores humanos: desenvolvimentos teóricos, metodológicos e aplicados. São Paulo: Senac. 2006. p. 397-431.

TAMAYO, A; GONDIM, M. G. C. Escala de valores organizacionais. Revista de Administração da Universidade de SãoPaulo (RAUSP), São Paulo, v. 31, n. 2, p. 62-72, abr./jun. 1996.

TAMAYO, A; MENDES, A. M.; PAZ, M. G. T. Inventário de valores organizacionais. Estudos de Psicologia, Campinas, v. 5, n. 2, p. 289-315, 2000.

TURNER, R.; HUEMANN, M.; KEEGAN, A. Human resource management in the projectoriented organization: employee well-being and ethical treatment. The International Journal of Project Management, Amsterdam, v. 26, n. 5, p. 577-585, 2008.

TZAFRIR, S. S. The relationship between trust, HRM practices and firm performance. The International Journal of Human Resource Management, Amsterdam, v. 16, n. 9, p. 1600$1622,2005$.

ULRICH, D. et al. Employee and customer attachment: synergies for competitive. Human Resource Planning, [s.1.], v. 14, n. 2, p. 89-102, 1991.

UYSAL, G. For the development of effective HRM systems: inter-relationships between HRM practices using correlation analysis. World Review of Entrepreneurship, Management and Sustainable Development, Olney, v. 8, n. 1, p. 1-12, 2012.

VAKOLA, M.; SODERQUIST, K. E.; PRATASCOS, G. P. Competence management in support of organizational change. International Journal of Manpower, Bingley, v. 28, n. 3/4, p. 260-275, 2007.

VEIGA, H. M. S. Comportamento Pró-ativo: relações com valores organizacionais, estímulos e barreiras à criatividade nas organizações e normas sociais. Tese (Doutorado) Instituto de Psicologia, Universidade de Brasília, Brasília, 2010.

WOOD JR., T.; TONELLI, M. J.; COOKE, B. Colonização e neo-colonização da gestão de recursos humanos no Brasil (1950-2010). Revista de Administração de Empresas, São Paulo, v. 51, n. 3, p. 232-243, 2011. 